Acta Crystallographica Section E

Structure Reports

Online

ISSN 1600-5368

\title{
2-Carboxypyridinium hydrogen chloranilate. Corrigendum
}

\section{Youhei Tabuchi, ${ }^{a}$ Akiko Takahashi, ${ }^{a}$ Kazuma Gotoh, Haruo Akashi ${ }^{\mathrm{b}}$ and Hiroyuki Ishida ${ }^{\mathrm{a} *}$}

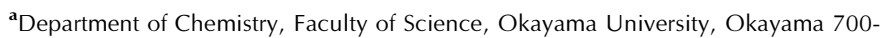
8530, Japan, and ${ }^{\mathbf{b}}$ Research Institute of Natural Sciences, Okayama University of Science, Okayama 700-0005, Japan

Correspondence e-mail: ishidah@cc.okayama-u.ac.jp

Received 7 February 2009; accepted 8 February 2009

The title and the chemical names of the paper by Tabuchi, Takahashi, Gotoh, Akashi \& Ishida [Acta Cryst. (2005), E61, o4215-o4217] are corrected.

In the paper by Tabuchi, Takahashi, Gotoh, Akashi \& Ishida [Acta Cryst. (2005), E61, 04215-04217], the title and the chemical names are incorrect with regard to the position of the carboxy group. The correct title of the original paper should be '3-Carboxypyridinium hydrogen chloranilate' and the chemical names 2-carboxypyridine and 2-carboxypyridinium in the Abstract should be 3-carboxypyridine and 3-carboxypyridinium, respectively. 